\title{
The number, dominance relationships and frequencies of self-incompatibility alleles in a natural population of Sinapis arvensis L. in South Wales
}

\author{
J.P. Stevens and \\ Q. O. N. Kay
}

School of Biological Sciences, University College of Swansea, Singleton Park, Swansea SA2 8PP, U.K.

The incompatibility genotypes of a further 25 plants from the South Wales population of Sinapis arvensis studied by Ford and Kay (1985) have been determined, and an additional $21 \mathrm{~S}$-alleles have been found. This brings the overall number of plants analysed from the population to 35 , and the total number of $\mathrm{S}$-alleles found also to 35 . It is estimated that there are $52 \mathrm{~S}$-alleles in the population.

Dominance interactions between S-alleles have been established in a total of 42 different heterozygotes (pooling data obtained during the present study with that of Ford and Kay (loc. cit.)). Co-dominance of alleles in both pollen and stigma occurred in 18 (42.8 per cent), dominance of one allele in the pollen occurred in 21 ( 50 per cent), dominance of one allele in the stigma occurred in two ( 4.8 per cent), and dominance of one allele in both pollen and stigma occurred in one ( 2.4 per cent). There is a minimum of three levels in the pollen dominance hierarchy, and two in the stigma dominance hierarchy; dominance interactions between $\mathbf{S}$-alleles in the stigma may be non-linear. There is some indication that pollen dominant alleles tend to be found in lower frequencies than pollen recessive alleles.

Preliminary data show that at least some $\mathbf{S}$-alleles in $\boldsymbol{S}$. arvensis have a very wide geographical distribution.

\section{INTRODUCTION}

It is well known that single-locus, multi-allelic self-incompatibility polymorphisms are subject to frequency-dependent selection. Two fundamental consequences of this type of selection are that (i) the potential number of different $\mathrm{S}$-alleles that can be maintained in a population is large, and (ii) the equilibrium frequency of each of the $k$ S-alleles in a population is expected to be $1 / k$, provided that all alleles are fully expressed in both pollen and stigma (as in all known gametophytic systems), and that there is no selection on the S-locus other than that associated with incompatibility (Wright, 1939; Campbell and Lawrence, 1981a).

If dominance interactions occur between $\mathrm{S}$ alleles (as in all known sporophytic systems), allelic equilibrium frequencies are expected to be modified by two factors. These are firstly the "recessive effect" (Sampson, 1974; also described by Bateman, 1952; Imrie et al., 1972), which tends to increase the frequency of recessive alleles, and secondly the "small number effect" (Sampson, 1974), which favours alleles at uncommon dominance levels (i.e., dominance levels represented by relatively few alleles). The underlying cause of these two effects is that recessive alleles, and alleles at uncommon dominance levels, tend to be involved in relatively few incompatible cross-pollinations. For recessive alleles, this is partly because they may occur as homozygotes, which are crosscompatible with more genotypes than are heterozygotes in which there is co-dominance, and partly because the expression of a recessive is wholly or partially masked when in heterozygous combination with a dominant allele. For alleles at uncommon dominance levels, on the other hand, it appears to be their occurrence in relatively few co-dominant heterozygous combinations (and consequently in relatively many in which there is dominance) that matters. This is because when there is dominance and only one allele is expressed, fewer incompatible cross-pollinations occur than when there is co-dominance and both alleles are expressed. It should be noted, however, that as the number of S-alleles in a population increases, the expected frequency of S-allele homozygotes approaches zero. This diminishes the influence of the recessive effect. Moreover, the more alleles that are present in a population, the lower the frequency of cross-pollinations between plants sharing an $\mathrm{S}$-allele. This reduces the influence of both the 
recessive effect and the small number effect (Sampson, 1974).

Despite this relatively strong theoretical background, we have very little information about the population genetics of sporophytic multi-allelic self-incompatibility polymorphisms in natural populations of wild species. Raphanus raphanistrum (Brassicaceae) constitutes the most thoroughly studied example to date. Sampson (1967) estimated that between 25 and 34 S-alleles (with upper and lower 95 per cent confidence limits of 15 and 82) exist in this species, and found some tentative evidence that recessive $\mathrm{S}$-alleles may occur at higher frequencies than dominant $\mathrm{S}$ alleles. In addition, Bateman (1954) made an approximate minimum estimate for the number of $\mathrm{S}$-alleles in a single natural population of Iberis amara (Brassicaceae) of 22, but investigated neither the frequencies of individual alleles nor their dominance relationships.

Sinapis arvensis L. has a sporophytic system of self-incompatibility that is controlled by many alleles at a single locus (Ford and Kay, 1985; Stevens and Kay, 1988). Our aim during the present study was to identify the S-allele genotypes of additional plants from the South Wales population of $S$. arvensis that was the subject of a previous investigation (Ford and Kay, loc. cit.). By increasing the sample size, we hoped to obtain a more reliable estimate of the number of S-alleles in the population, to begin to elucidate the S-allele dominance hierarchy, and to investigate the relationship between $S$-allele position in the dominance hierarchy and S-allele frequency in the population sample. In addition, analysis of the S-allele constitutions of two geographically remote populations was commenced.

\section{MATERIALS AND METHODS}

In January 1986, three seeds were sown from samples collected from each of 32 randomly chosen plants from the South Wales population of S. arvensis studied by Ford and Kay (1985) (grid reference $\mathrm{SS} 474.925$ ). In addition, three seeds were sown from samples collected from each of nine plants from a population near Little Steeping, Lincolnshire, England (TF 440.609) (which is approximately $350 \mathrm{~km}$ distant from the South Wales site), and from each of two plants from a population near Koxares, Crete. (The seed samples from the South Wales population were collected in October 1981 simultaneously with the collection of the sample of adult plants for the study described by Ford and Kay (loc. cit.).) All three populations were large (each consisting of several hundred plants), and grew as weeds of arable fields.

One seedling from each triplet was randomly selected and raised to flowering. These plants constituted the parental (or $I_{0}$ ) generation. They were all selfed by bud-pollination to produce seed for the first inbred (or $\mathrm{I}_{1}$ ) generation, and selfed in the open flower to determine the strength of the incompatibility reaction. In addition, all the plants, except for ten from the South Wales population, were cross-pollinated in a full diallel and their compatibility reactions determined, so that plants sharing dominant or co-dominant alleles could be identified.

$I_{1}$ families were raised for 27 of the 32 plants from the South Wales population, both of the two plants from Crete, and one of the nine plants from the Lincolnshire population. Each $\mathrm{I}_{1}$ family consisted of a minimum of 19 full sibs, which, assuming that the parent was heterozygous at the S-locus, gave a probability of 0.99 that both S-allele homozygotes would be included (see Mackay, 1977). Four to six families were raised at a time, with intervals of six to eight weeks between successive sowings. This generally provided sufficient time to complete the pollinations within each batch of families before the next batch came into flower. Whenever possible, the families of plants that were known from the $I_{0}$ diallel to share an allele in common were grown up together. Tester plants for the 14 S-alleles identified previously by Ford and Kay (1985) were raised simultaneously with the $I_{1}$ families.

Within each $I_{1}$ family, successive chains of cross-pollinations were made between sibs, and the compatibility reactions determined. The results from these crosses were analysed following Wallace $(1979 b)$. This procedure enables the incompatibility genotype of each $I_{1}$ plant to be established and the type of S-allele dominance interaction determined. In one $I_{1}$ family, only two, rather than three, incompatibility groups segregated; for this $I_{0}$ plant, a second inbred $\left(I_{2}\right)$ generation was required to confirm dominance of one $\mathrm{S}$-allele in both pollen and stigma, and to isolate the dominant homozygote. When two or more $I_{1}$ families raised simultaneously were known to share an allele in common, the S-allele homozygotes isolated from one family were used to identify genotypes in the other(s).

Once both S-allele homozygotes had been isolated from an $I_{1}$ (or $I_{2}$ ) family, their identities were checked by crossing with the tester plants for $S_{1}$ 
to $S_{14}$, and with further tester plants for newly identified recessive $\mathrm{S}$-alleles.

New S-alleles were numbered consecutively, beginning with $\mathrm{S}_{15}$, and each was incorporated into the $\mathrm{S}$-allele seed collection of $S$. arvensis.

The conditions under which plants were cultivated, and the methods used for pollinating and determining compatibility reactions are described elsewhere (Stevens and Kay, 1988).

\section{RESULTS}

\section{The South Wales population}

Number of $S$-alleles found. The incompatibility genotypes of 25 of the 27 plants for which $I_{1}$ families were raised were successfully determined. (In one $\mathrm{I}_{1}$ family the plants died before all pollinations had been completed, and in another a high degree of self- and cross-compatibility among sibs made interpretation of pollinations impossible; the reasons for the breakdown of self-incompatibility in the latter family are not clear.) None of the 25 $\mathrm{I}_{0}$ plants successfully analysed was homozygous at the S-locus. A total of 21 new S-alleles was found, and these were allocated numbers from $S_{15}$ to $\mathrm{S}_{35}$.

If we combine these results with those of the previous study (Ford and Kay, 1985), which is appropriate because the population samples for both investigations were collected on the same occasion, a total of 35 plants have been examined from the South Wales population, and 35 different $\mathrm{S}$-alleles have been found (table 1).

Some indication of the completeness of sampling in a survey of this nature can be deduced from a repeatability statistic $(R)$ (Campbell and Lawrence, 1981 $a$ ), which has values of between 0 and 1 ; the closer $R$ is to 1 , the more thorough the study. With single-locus multi-allelic gametophytic systems, $R=1-(n-3) /(m-3)$, where $n$ is the number of different alleles found in a sample of size $m$ copies of the S-gene examined; 3 is subtracted from both $m$ and $n$ because a minimum of 3 $\mathrm{S}$-alleles is required for a population to persist (Campbell and Lawrence, 1981a). With singlelocus sporophytic systems, a population having only $2 \mathrm{~S}$-alleles (one dominant and one recessive) can persist, so it would seem appropriate to modify the statistic to $R=1-(n-2) /(m-2)$. Thus, for the two studies of $S$. arvensis combined, $R=$ $1-(35-2) /(70-2)=0 \cdot 51$. This moderately low value of $R$ is a consequence of the large number of different alleles that occurred in the sample, and suggests that substantially more alleles remain to be found in the population. Nevertheless, our study of $S$. arvensis appears to constitute the most extensive of its kind in a non-cultivated species with a sporophytic, multi-allelic system of self-incompatibility.

$S$-allele dominance interactions. Dominance interactions have been determined in 42 different heterozygous combinations of S-alleles ( 32 during this study and 10 during the previous study by Ford and Kay (1985)). Co-dominance of S-alleles in both pollen and stigma occurred in 18 heterozygotes ( 42.8 per cent), dominance of one $S$-allele in the pollen (with co-dominance in the stigma) in 21 ( 50 per cent), dominance of one S-allele in the stigma (with co-dominance in the pollen) in two $(4.8$ per cent), and dominance of one $S$-allele in both pollen and stigma in one ( 2.4 per cent).

On the basis of this information, the S-alleles may be arranged into provisional dominance hierarchies (fig. 1). In the stigma, two dominance levels were apparent, but there was evidence of

Table 1 S-allele composition of a sample of 35 plants of Sinapis arvensis from a natural population in South Wales. Analysis of plants 1 to 10 was carried out by Ford and Kay (1985), and of plants 11 to 35 by the present authors. S-alleles marked with a dot above are dominant in the pollen, and S-alleles underlined are dominant in the stigma

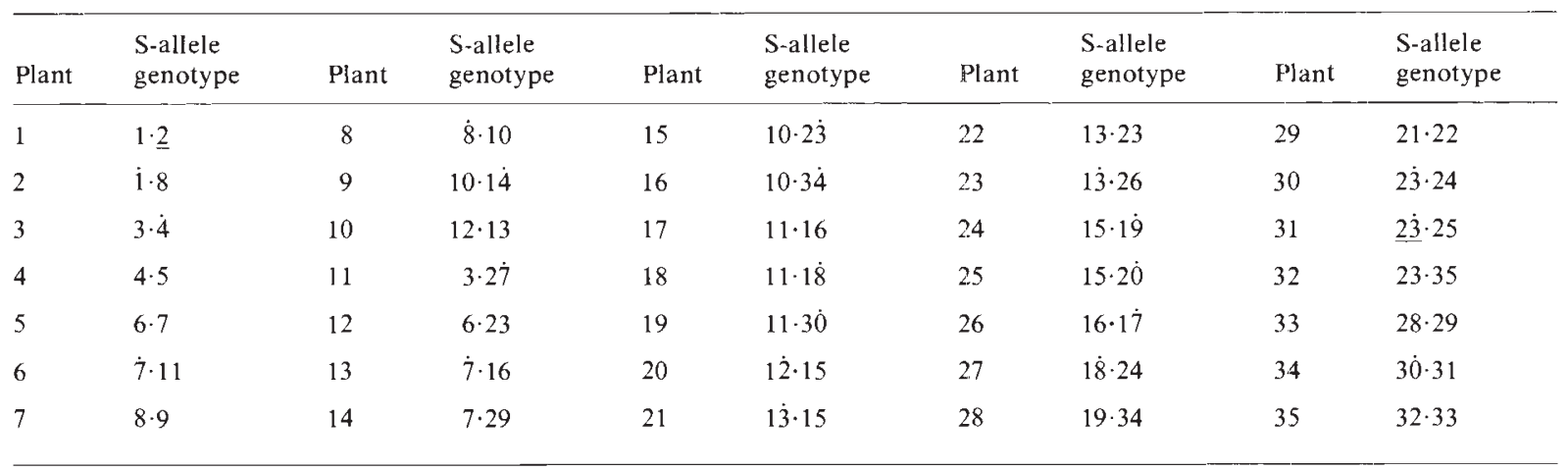



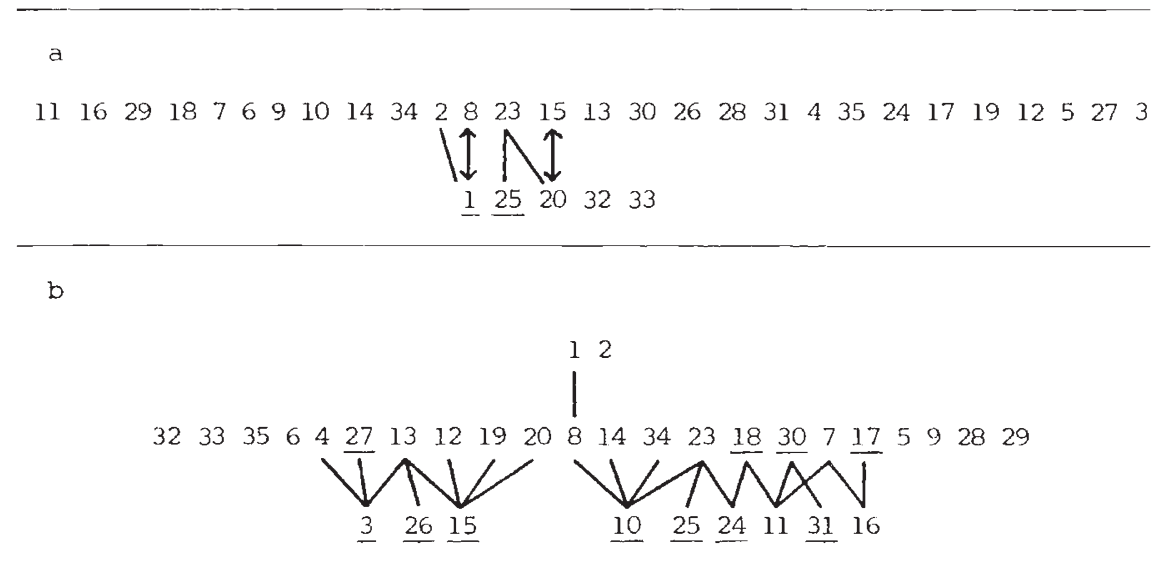

Figure 1 S-allele dominance hierarchy in the stigma (a) and in the pollen (b) (based on combined data from the present study and Ford and Kay (1985)). The numbers identify S-alleles. A plain line connecting two numbers indicates that the upper is known to be dominant to the lower, while a double-headed arrow indicates co-dominance between the two alleles (used when interactions are non-linear). All alleles are co-dominant with at least one other allele on their level, apart from alleles that are underlined, which were not observed in any co-dominant combinations. It is possible that the latter may have intermediate levels of dominance.

non-linear relationships between some of the alleles (fig. 1a). Consequently, exact positions of alleles in the stigma dominance hierarchy cannot yet be established. In the pollen, three dominance levels were apparent and no non-linear relationships were found (fig. $1 b$ ). It should be mentioned, however, that certain alleles (which are underlined in fig. 1) occurred as a dominant or as a recessive but were not observed in any co-dominant combinations; it is possible that at least some of these alleles have intermediate levels of dominance. In addition, two of the 35 S-alleles could not be assigned a place in either hierarchy. These are $S_{21}$ and $S_{22}$, which were observed in a single heterozygous combination (with each other), in which both alleles were co-dominant in pollen and stigma.

$S$-allele frequencies. S-allele frequencies in the South Wales population sample are shown in fig. 2 . The majority of alleles occurred in the sample once or twice; the most frequently detected allele occurred six times.

The data on S-allele dominance is too fragmentary to warrant statistical tests for evidence of the recessive and small number effects. However, there appears to be a tendency for pollen dominant alleles to occur at lower frequencies than pollen recessive alleles, the mean frequencies of alleles at level 1 (top dominant) level 2 (middle dominant) and level 3 (recessive) being $1.50,1.95$ and 2.44 respectively. This trend is in agreement with that predicted by the recessive effect.
Modification of $S$-allele expression. The $32 \mathrm{I}_{0}$ plants from the South Wales population were all fully self-incompatible. However, in five of the $25 I_{1}$

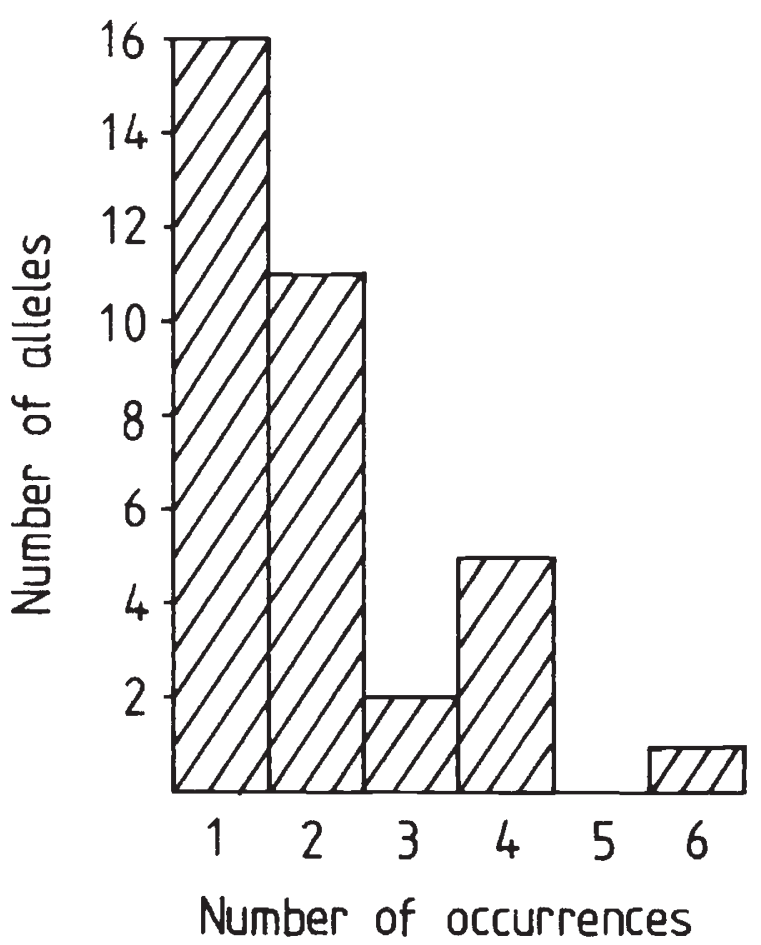

Figure 2 Frequency distribution of S-alleles in a sample of 35 plants of Sinapis arvensis from a natural population in South Wales (based on combined data from the present study and Ford and Kay (1985)). 
families successfully analysed, S-allele expression was occasionally modified in some plants. Weakening of the expression of $\mathrm{S}_{27}$ in the pollen and of both alleles in the stigma occurred in some $\mathrm{S}_{3.27}$ heterozygotes, and weakening of the expression of $\mathrm{S}_{15}$ occurred in the pollen of some $\mathrm{S}_{12.15}$ plants. Partial (or incomplete) dominance was occasionally observed in some $S_{13.15}$ heterozygotes $\left(S_{15}\right.$ partially dominant in the stigma), in some $S_{13.23}$ heterozygotes $\left(S_{13}\right.$ partially dominant in the pollen, and $S_{23}$ partially dominant in the stigma), and in some $S_{32.33}$ heterozygotes $\left(S_{33}\right.$ partially dominant in both pollen and stigma). These modifications result in what are normally incompatible reactions (including both selfs and crosses) being "leaky"; instead of a complete absence of pollen tubes in the style, a small number occur, but far fewer than are found following a compatible pollination. The fact that these abnormalities were only observed in inbred $\left(I_{1}\right)$ plants suggests that they may have been caused by recessive alleles segregating at modifier loci. It is well known that factors other than the precise specificity of an S-allele may modify its expression (e.g., Wallace, 1979 a; Visser et al., 1982).

\section{The geographical distribution of S-alleles}

The two plants from Crete, and the one from Lincolnshire for which $\mathrm{I}_{1}$ families were raised, each contained one S-allele that had already been found in the South Wales population $\left(S_{20}, S_{20}\right.$ and $S_{32}$ respectively) and one new $\mathrm{S}$-allele $\left(\mathrm{S}_{36}, \mathrm{~S}_{37}\right.$ and $S_{38}$ respectively). (Details of the $I_{1}$ families of these three plants (KX13, KX17, LS12) have been given elsewhere (Stevens and Kay, 1988).) From these results it is clear that at least some S-alleles in $S$. arvensis have a very wide geographical distribution.

The number of dominant or co-dominant alleles that are shared between the South Wales population sample and the remaining eight plants from Lincolnshire (for which $\mathrm{I}_{1}$ families were not raised) can be determined from the $\mathrm{I}_{0}$ diallel. In this diallel, 17 alleles were fully expressed in both pollen and stigma (i.e., were dominant or codominant) in the 20 plants from the South Wales population that were included and whose S-allele genotypes were subsequently determined. Of these 17 alleles, four were picked up more than once in the 20 South Wales plants, and an equal number were shared between plants from the two localities. This preliminary analysis suggests that the level of divergence between these two geographically remote British populations in their S-allele constitutions might not be great.

\section{DISCUSSION}

It is of considerable interest to make inferences about the total number of S-alleles $(N)$ in the South Wales population of $S$. arvensis from the random sample of 35 plants analysed. However, the three basic methods that are available for making such inferences (Bateman, 1947; Whitehouse, 1949; Paxman, 1963) were all developed for use with gametophytic systems and they make two assumptions that are not strictly satisfied by sporophytic systems. The first of these assumptions is that the two incompatibility alleles within a diploid individual must be different. In sporophytic systems, homozygotes for recessive $\mathrm{S}$-alleles can occur. In the South Wales population of $S$. arvensis, however, the frequency of S-allele homozygotes is likely to be very low, because of the large number of alleles present, each at a low frequency. Therefore, it would seem that if one of these methods of estimating $N$ were applied to our results, the technical violation of the first assumption would be unlikely to result in seriously misleading conclusions. The second assumption is that genotype frequencies within the population are equal. If S-allele frequencies are not equal, Bateman's method breaks down and the methods of Whitehouse and Paxman yield estimates of $N$ that are biased downwards (O'Donnell and Lawrence, 1984). There are insufficient data for the South Wales population of $S$. arvensis to justify a statistical test of equality of allele frequencies, but in sporophytic multi-allelic systems in general, Sallele frequencies at equilibrium are expected to be unequal. The problem of estimating $N$ when allele frequencies are unequal has been considered by O'Donnell and Lawrence (1984). They developed and tested two modifications $\left(E_{1}\right.$ and $E_{2}$ ) of the maximum likelihood method of Paxman (1963), one of which $\left(E_{2}\right)$ gave reasonably accurate, though still somewhat corsservative, estimates of $N$ when allele frequencies were unequal. This method would, therefore, seem the most appropriate to apply to the $S$. arvensis data. The estimate for $N$ yielded by $E_{2}$ is 52 . (For comparison, Paxman's maximum likelihood method, assuming equal genotype frequencies, gave $N=43$ with 99 per cent confidence limits of 35 and 60 .)

This estimate of $N(52)$ is considerably larger than the estimates that have been made for $R$. 
raphanistrum of 25 to $34 \mathrm{~S}$-alleles in the entire species (Sampson, 1967) and for I. amara of 22 S-alleles in a single locality (Bateman, 1954). Our estimate for $S$. arvensis is more comparable with figures for Papaver rhoeas, which has a gametophytic multi-allelic system; in each of three large populations in the West Midlands, surveyed by Lawrence and O'Donnell (1981) and Campbell and Lawrence $(1981 b)$, at least 40 different Salleles, but probably not many more than this number, were estimated to occur (O'Donnell and Lawrence, 1984). In Brassica oleracea, a cultivated species with a sporophytic multi-allelic self-incompatibility system, between 18 and 35 different Salleles have been found in each of four different varieties (reviewed by Ockendon, 1985). As expected, considerable overlap occurs between varieties in their S-allele constitutions (due to their recent common ancestry), but some differentiation has occurred, and the total number of different Salleles found in the four varieties combined is 49 (Ockendon, 1985). This is quite a large number bearing in mind that artificial selection and breeding is likely to have depleted the S-allele pool (Ockendon and Currah, 1979; Ockendon, 1974; 1982).

The pattern of dominance interactions found so far between S-alleles in $S$. arvensis is broadly similar to that found in $R$. raphanistrum (Sampson, 1964). Dominance, in either pollen or stigma, is about as common as co-dominance in both; dominance in the pollen is more frequent than dominance in the stigma; and as yet non-linear interactions have not been found in the pollen. In $B$. campestris, there is a higher frequency of dominance in the stigma (Mackay, 1977), and in B. oleracea, non-linear dominance interactions have been observed in both pollen and stigma (Thompson and Taylor, 1966).

In gametophytic self-incompatibility systems, all alleles are expected to occur in approximately equal frequencies at equilibrium; a test for equilibrium may, therefore, be made by comparing observed allele frequencies with the expected frequencies based on this null hypothesis (see Campbell and Lawrence, 1981b). With sporophytic multi-allelic self-incompatibility, the situation is more complex. It is expected that $\mathrm{S}$-allele equilibrium frequencies will be unequal, but the extent and type of this inequality cannot be calculated unless the full range of dominance interactions is known. In $S$. arvensis, the dominance interactions in 42 different heterozygous combinations have been determined and a preliminary picture of the S-allele dominance hierarchy obtained, but the information is not sufficient for the calculation of expected S-allele equilibria.

Some evidence has been found that pollen dominant alleles occur at lower frequencies than pollen recessive alleles in natural populations of $R$. raphanistrum (Sampson, 1967) and in cultivars of B. oleracea (Ockendon, 1974, 1980, 1982). A similar trend was found in the South Wales population of $S$. arvensis.

A final point concerns the geographical distribution of S-alleles in S. arvensis. Although we have only a limited amount of information on the Sallele constitutions of the Lincolnshire and Cretan populations, it is already apparent that at least some $\mathrm{S}$-alleles have a very wide geographical distribution. This has also been found in $R$. raphanistrum in which five $\mathrm{S}$-alleles occurred in small samples of plants from both Poland and Canada (Sampson, 1967). In P. rhoeas, three isolated populations in the English West Midlands, known to vary in a number of metrical characters, were not found to differ significantly in their S-allele constitutions (Campbell and Lawrence, $1981 a$; Lawrence and O'Donnell, 1981). Further research on S. arvensis is now required to determine more fully the extent to which the S-allele pools in geographically remote populations have differentiated.

Acknowledgements We are indebted to David P. Stevens for writing the Fortran program used to calculate the 99 per cent confidence interval for the maximum likelihood estimate of $N$, to Martin A. Ford for initiating the S-allele seed collection (for $S_{1}$ to $S_{14}$ ) and for collecting the seed samples from the Lincolnshire population, and to both for valuable discussion throughout the project and comments on the manuscript. We are also grateful to Michael J. Lawrence and David J. Ockendon for comments on an earlier draft of the manuscript. We wish to thank the N.E.R.C. for financial support.

\section{REFERENCES}

BATEMAN, A. J. 1947. Number of S-alleles in a population. Nature, 160, 337.

BATEMAN, A, J. 1952. Self-incompatibility systems in angiosperms. I. Theory. Heredity, 6, 285-310.

BATEMAN, A. J. 1954. Self-incompatibility systems in angiosperms. II. Iberis amara. Heredity, 8, 305-332.

CAMPBELL, J. M. AND LAWRENCE, M. J. $1981 a$. The population genetics of the self-incompatibility polymorphism in Papaver rhoeas. I. The number and distribution of S-alleles in families from three localitities. Heredity, 46, 69-79.

CAMPBELL, J. M. AND LAWRENCE, M. J. $1981 b$. The population genetics of the self-incompatibility polymorphism in Papaver rhoeas. II. The number and frequency of S-alleles in a natural population (R106). Heredity, 46, 81-90.

FORD, M. A. AND KAY, Q. O. N. 1985. The genetics of incompatibility in Sinapis arvensis L. Heredity, 54, 99-102. 
IMRIE, B. C., KIRKMAN, C. T. AND ROSS, D. R. 1972. Computer simulation of a sporophytic incompatibility breeding system. Aust. J. Biol. Sci., 25, 343-349.

LAWRENCE, M. J. AND O'DONNELL, S. 1981. The population genetics of the self-incompatibility polymorphism in Papaver rhoeas. III. The number and frequency of S-alleles in two further natural populations (R102 and R104). Heredity, 47, 53-62.

MACKAY, G. R. 1977. A diallel cross method for the recognition of $\mathrm{S}$ allele homozygotes in turnip, Brassica campestris L. ssp. rapifera. Heredity, 38, 201-208.

OCKENDON, D. J. 1974. Distribution of self-incompatibility alleles and breeding structure of open-pollinated cultivars of Brussels sprouts. Heredity, 33, 159-171.

OCKENDON, D. J. 1980. Distribution of S-alleles and breeding structure of Cape broccoli (Brassica oleracea var. 'italica'). Theor. Appl. Genet., 58, 11-15.

OCKENDON, D. J. 1982. An S-allele survey of cabbage (Brassica oleracea var. capitata). Euphytica, 31, 325-331.

OCKENDON, D. J. 1985. Genetics and physiology of self-incompatibility in Brassica. In Sussex I., Ellingboe, A., Crouch, M. and Malmberg, R. (eds) Plant Cell/Cell Interactions (Current Communications in Molecular Biology Ser.), Cold Spring Harbor, pp 1-6.

OCKENDON, D. J. AND CURRAH, L. 1979. Breeding structure of a highly selected cultivar of cabbage (Brassica oleracea var. capitata). Heredity, 42, 373-379.

O'DONNELL, S. AND LAWRENCE, M. J. 1984. The population genetics of the self-incompatibility polymorphism in Papaver rhoeas. IV. The estimation of the number of alleles in a population. Heredity, 53, 495-507.
PAXMAN, G. J. 1963. The maximum likelihood estimation of the number of self-sterility alleles in a population. Genetics, 48, 1029-1032.

SAMPSON, D. R. 1964. A one-locus self-incompatibility system in Raphanus raphanistrum. Can. J. Genet. Cytol., 6, 435-445.

SAMPSON, D. R. 1967. Frequency and distribution of self-incompatibility alleles in Raphanus raphanistrum. Genetics. 56, 241-251.

SAMPSON, D. R. 1974. Equilibrium frequencies of sporophytic self-incompatibility alleles. Can. J. Genet. Cytol., 16, 611618.

STEVENS, J. P. AND KAY, Q. O. N. 1988. The number of loci controlling the sporophytic self-incompatibility system in Sinapis arvensis L. Heredity, 61, 411-418.

THOMPSON, K. F. AND TAYLOR, J. P. 1966. Non-linear dominance relationships between S-alleles. Heredity, 21, 345-362.

VISSER, D. L., VAN HAL, J. G. AND VERHOEVEN, W. H. 1982. Classification of $\mathrm{S}$-alleles by their activity in S-heterozygotes of Brussels sprouts (Brassica oleracea var. gemmifera (DC.) Schultz). Euphytica, 31, 603-611.

WALLACE, D. H. $1979 a$. Interactions of $\mathrm{S}$ alleles in sporophytically controlled self incompatibility of Brassica. Theor. Appl. Genet., 54, 193-201.

Wallace, D. H. 1979b. Procedures for identifying S-allele genotypes of Brassica. Theor. Appl. Genet., 54, 249-265.

WHITEHOUSE, H. L. K. 1949. Multiple-allelomorph heterothallism in the fungi. New Phytol., 48, 212-244.

WRIGHT, S. 1939. The distribution of self-sterility alleles in populations. Genetics, 24, 538-552. 